Edith Cowan University

Research Online

ECU Publications Post 2013

2015

Effective, clinically feasible and sustainable: Key design features

of psycho-educational and supportive care interventions to

promote individualised self-management in cancer care

P. Schofield

Suzanne Chambers

Edith Cowan University

Follow this and additional works at: https://ro.ecu.edu.au/ecuworkspost2013

Part of the Oncology Commons

10.3109/0284186X.2015.1010016

Schofield, P., Chambers, S. (2015). Effective, clinically feasible and sustainable: Key design features of psychoeducational and supportive care interventions to promote individualised self-management in cancer care in Acta Oncologica, 54(5), 805-812. Available here. This Journal Article is posted at Research Online. https://ro.ecu.edu.au/ecuworkspost2013/1210 


\section{Effective, clinically feasible and sustainable: Key design features of psycho-educational and supportive care interventions to promote individualised self-management in cancer care}

\section{Penelope Schofield \& Suzanne Chambers}

To cite this article: Penelope Schofield \& Suzanne Chambers (2015) Effective, clinically feasible and sustainable: Key design features of psycho-educational and supportive care interventions to promote individualised self-management in cancer care, Acta Oncologica, 54:5, 805-812, DOI: 10.3109/0284186X.2015.1010016

To link to this article: http://dx.doi.org/10.3109/0284186X.2015.1010016

Published online: 27 Mar 2015.

Submit your article to this journal

Џ Article views: 904

Q View related articles $₫$

View Crossmark data $\nearrow$

Citing articles: 5 View citing articles 


\title{
Effective, clinically feasible and sustainable: Key design features of psycho-educational and supportive care interventions to promote individualised self-management in cancer care
}

\author{
PENELOPE SCHOFIELD ${ }^{1,2,3,4,5}$ \& SUZANNE CHAMBERS CH,7,8,9,10 $^{6}$ \\ ${ }^{1}$ Department of Psychology, Swinburne University of Technology, Melbourne, Victoria, Australia, \\ ${ }^{2}$ Department of Cancer Experiences Research, Peter MacCallum Cancer Centre, Melbourne, Victoria, Australia, \\ ${ }^{3}$ Sir Peter MacCallum Department of Oncology, Faculty of Medicine, Dentistry and Health Sciences, The University \\ of Melbourne, Parkville, Victoria, Australia, ${ }^{4}$ School of Behavioural Science, The University of Melbourne, \\ Parkville, Victoria, Australia, ${ }^{5}$ School of Nursing, The University of Melbourne, Parkville, Victoria, Australia, \\ ${ }^{6}$ School of Applied Psychology, Griffith University, Brisbane, Queensland, Australia, ${ }^{7}$ Cancer Council Queensland, \\ Brisbane, Queensland, Australia, ${ }^{8}$ Prostate Cancer Foundation of Australia, Sydney, New South Wales, Australia, \\ ${ }^{9}$ Health $\mathcal{G}$ Wellness Institute, Edith Cowan University, Perth, Western Australia, Australia and ${ }^{10}$ Centre for Clinical \\ Research, University of Queensland, Queensland, Australia
}

\section{ABSTRACT}

As the global burden of cancer increases healthcare services will face increasing challenges in meet the complex needs of these patients, their families and the communities in which they live. This raises the question of how to meet patient need where direct clinical contact may be constrained or not readily available. Patients and families require resources and skills to manage their illness outside of the hospital setting within their own communities.

Aim. To propose a framework for the development and delivery of psycho-educational and supportive care interventions drawing on theoretical principles of behaviour change and evidence-based interventions, and based on extensive experience in developing and testing complex interventions in oncology.

Approach. At the core of this intervention framework are considerations of efficiency: interventions are designed to cater for individuals' unique needs; to place minimal demands on the health system infrastructure and to be rapidly disseminated into usual care if successful. There are seven key features: 1) Targeting cancer type and stage; 2) Tailoring to unique individual needs; 3) Promotion of patient self-management of their disease and treatment side effects; 4) Efficient delivery of the intervention; 5) Training and adherence to protocol; 6) Ensuring the intervention is evidencebased; 7) Confirming stakeholder acceptability of the intervention.

Application. A case study of a randomised controlled trial which tested psycho-educational oncology interventions using this framework is presented. These interventions were designed to cater for individuals' unique needs and promote self-management while placing minimal demands on the acute health care setting.

Discussion. Innovative ways to realise the potentially major impact that psycho-educational and supportive care interventions can have on psychological morbidity, coping, symptoms and quality of life in serious and chronic illness are needed. This framework, which is driven by theory, evidence, and experience, is designed to ensure that interventions are effective, clinically feasible and sustainable.

Cancer is the leading cause of burden of disease in the world, accounting for nearly one-fifth of the total disease burden [1]. The diagnosis and subsequent treatment of cancer is a major life stress that is followed by a range of well described psychological, social, physical, sexual, spiritual and practical difficulties [2]. The demand for oncology services in the
US was estimated to rise $48 \%$ between 2005 and 2020 [3]. In the face of mounting pressure on cancer care services, consumers expect better quality care than they are currently receiving.

With the global economic downturn affecting public spending and the spiralling costs of cancer care, healthcare services in developed nations are

Correspondence: P. E. Schofield, Department of Cancer Experiences Research, Peter MacCallum Cancer Centre, Locked bag 1, A'Beckett Street, Victoria 8006, Australia. E-mail: penelope.schofield@petermac.org. 
experiencing a period of economic restraint [4]. Consequently, it is highly unlikely that the rising demand for high quality oncology care will be met by increased direct clinical contact. As cancer incidence and prevalence increases, attention will focus more towards meeting the psychosocial care needs of patients and families [5]. As the number of those living with cancer is expected to rise [1], research is urgently required to realise the potentially major impact that psycho-educational and supportive care interventions can have on the delivery of high quality cancer care and improving patient-reported outcomes with minimal or no additional cost.

\section{Theoretical approaches to behaviour change}

The core consideration when designing effective psycho-educational or supportive care interventions is how to achieve long-term positive outcomes for cancer patients and carers by supporting them to adopt novel (or ceasing existing) behaviours to manage health concerns at home. This goal will also require behaviour change for the health professionals providing care to these patients and carers and for those whose role may include delivering or facilitating the intervention.

Self-efficacy is a key concept in behaviour change. Self-efficacy is defined as task-specific confidence, that is, a person's beliefs in their ability to succeed in a given task. The higher one's self-efficacy, the more likely one is to engage in a behaviour and succeed [6]. Theoretically, an individual's self-efficacy beliefs can be derived from four sources [6]. First, mastery experience is a reflection and analysis of one's actions in relation to the task. Second, social modelling refers to gaining self-efficacy through observing others succeed at the task. Third, self-efficacy can be promoted by the social persuasion or encouragement from others. Fourth, physiological and affective reactions, such as feeling calm and assured, can influence a person's confidence in achieving a task. Self-efficacy is an essential element of many behaviour change theories including the Theory of Planned Behaviour [7]; Social Cognitive theory [6], the Health Belief Model [8] and Trans-theoretical or Stages of Change model [9]. While these theories have different elements and specific mechanisms, they are all essentially social-cognitive models which comprise attitudinal beliefs, social influences and self-efficacy in relation to the behaviour. A meta-analysis of interventions aiming to improve quality of life in cancer patients found that interventions informed by social cognitive theory (SCT) larger effect sizes for overall quality of life, global affect, depression, physical and social outcomes, compared to those with few or no SCT components [10]. Hence, a consideration of how SCT informs intervention design and delivery is crucial.

House and Kahn [11] delineates three sources of social support which are important to consider in intervention design: instrumental, emotional and informational support. Social support may aid patient adjustment by acting as a stress buffer and/or as a coping resource [12]. As such, interventions that enhance social support either by strengthening existing networks or developing new adjunctive networks are desirable, and can often be mobilised at low cost. As an example, peer support interventions have organically emerged worldwide from communities themselves [13], presenting as an intervention resource that as yet has arguably been under-utilised in both research and formal healthcare service delivery.

Finally, motivation is now recognised as crucial to successful behaviour change. Self-Determination Theory [14] describes a framework of intrinsic motives and extrinsic motives or social conditions which either facilitate or forestall self-motivation in relation to behaviour change. Underpinned by Self-Determination Theory, Motivational Interviewing [15] is a client-centred method that allows patients to explore and resolve their own ambivalence about the behaviour change to evoke self-motivation and supports self-efficacy as opposed to traditional didactic health advice provision. Drawing upon these and other theories of behaviour change is likely to strengthen the intervention effectiveness.

\section{Targeting and tailoring}

There has been growing recognition that a "onesize" intervention does not fit all and that psychoeducational and supportive care interventions need to be targeted to the cancer type and stage, and tailored to the individual's needs and circumstances. Intuitively, screening for distress, unmet needs, uncontrolled symptoms or side effects is a necessary first step. Many advocate that routine screening for distress and unmet needs should be implemented as usual care within cancer treatment facilities [16]. Others maintain that it cannot be recommended until patient benefit and cost-effectiveness can be demonstrated $[17,18]$. To date, the results of randomised controlled trials of screening suggest that screening alone will not improve patient outcomes $[19,20]$. Rather, after patients with potential needs have been identified, further assessment and triage with referral to evidence-based management is needed $[19,20]$. Of high relevance here is that a sizable proportion of patients identified as having high distress or needs decline specialised psychosocial or supportive care services [21,22], most of whom do so based on a preference to manage their own 
distress [23]. This highlights the importance of effective self-management as a key intervention approach.

\section{Self-management}

Self-management of chronic diseases includes activities undertaken by the patient to manage their disease or side effects. Self-management is distinct from but complements traditional patient education by emphasising patient control over their disease and quality of life [24]. With the traditional education, the patient receives disease information and advice about technical skills from a healthcare professional [24]. In contrast, Lorig and colleagues [24-26] posit that there are two essential elements of self-management education: 1) patients selfidentify their most important problems and learn to solve these problems, that is, develop problem solving skills; and 2) these skills are used to address three tasks associated with chronic disease: medical management; social role management; and emotional management. The core self-management skills are: 1) defining the problem and generating possible solutions; 2) making informed decision based on sufficient and appropriate information; 3) locating and using reputable resources; 4) forming collaborative partnerships with healthcare providers providing knowledge about the disease and patient providing knowledge about their life, values and preferences; 5) taking action to implement the action plan to solve the problem; 6) feeling confident in one's ability to accomplish the action plan. Internal motivation and high self-efficacy underpin the successful development of these skills.

Self-management approaches are critically important to this framework not only because evidence suggests that they are more effective than information provision alone but it has the potential to increase the intensity of the intervention while reducing the healthcare costs [26].

\section{Intervention intensity}

A recent meta-analysis demonstrates that small to medium effects were observed for either individual or group psychotherapy and psycho-education [27]. Those studies which used samples of patients reporting high distress initially demonstrated larger effects and the longer the intervention produced more sustained effects. This meta-analysis points to the importance of defining and identifying who may benefit from intervention; what the content of this intervention should be and what intensity or "dose" is required to achieve a clinically significant shift in the key outcome measure(s). The goal here is to deliver what is needed where it is needed.
This provides a clinical and public health imperative to identify patients and family members experiencing high psychological, social, physical, sexual, spiritual or practical difficulties; and provides coaching in self-management or referrals to services that match their needs. Accessible, clinically feasible, effective interventions targeted to cancer type and tailored to individual needs are needed.

\section{Aim}

The aim of this paper is to propose a psychoeducational intervention framework. This framework will address the demands associated with a particular type of cancer and stage; the personal context of the individual and their personal needs and circumstances; their family and social setting; is based on theoretical principles of behaviour change and selfmanagement education in evidence-based strategies; is acceptable to patients, families and oncology health professionals and takes into account the clinical feasibility and infrastructure needed to deliver the intervention for translation to the population.

\section{Approach}

Through our program of research, we have developed a theoretically and empirically driven framework to the development and delivery of psycho-educational and supportive care interventions to ensure that they are effective, feasible and sustainable now and into the future.

This intervention framework takes into account the heterogeneity of patients' needs by considering clinical differences associated with the cancer and point in illness trajectory; personal variations of the individual, their cultural background, social circumstances and unique needs; and an appreciation of differing intensities of patient and carer need. At the core of this framework are considerations of efficiency: interventions are designed to place minimal demands on the infrastructure needed to deliver the intervention for translation to the population, and we propose that these considerations have widespread application in both low and high resource communities.

In order for an intervention to be effective, and easily translated into usual care practice, we argue that a number of key features are required. These are represented in Figure 1.

Targeting cancer-related needs. Prior to embarking on a program of intervention research, it is necessary to understand the nature of the problems faced by each group so that the support provided is relevant to patient concerns. Characteristics of the cancer type, stage or treatment or point in the illness trajectory 


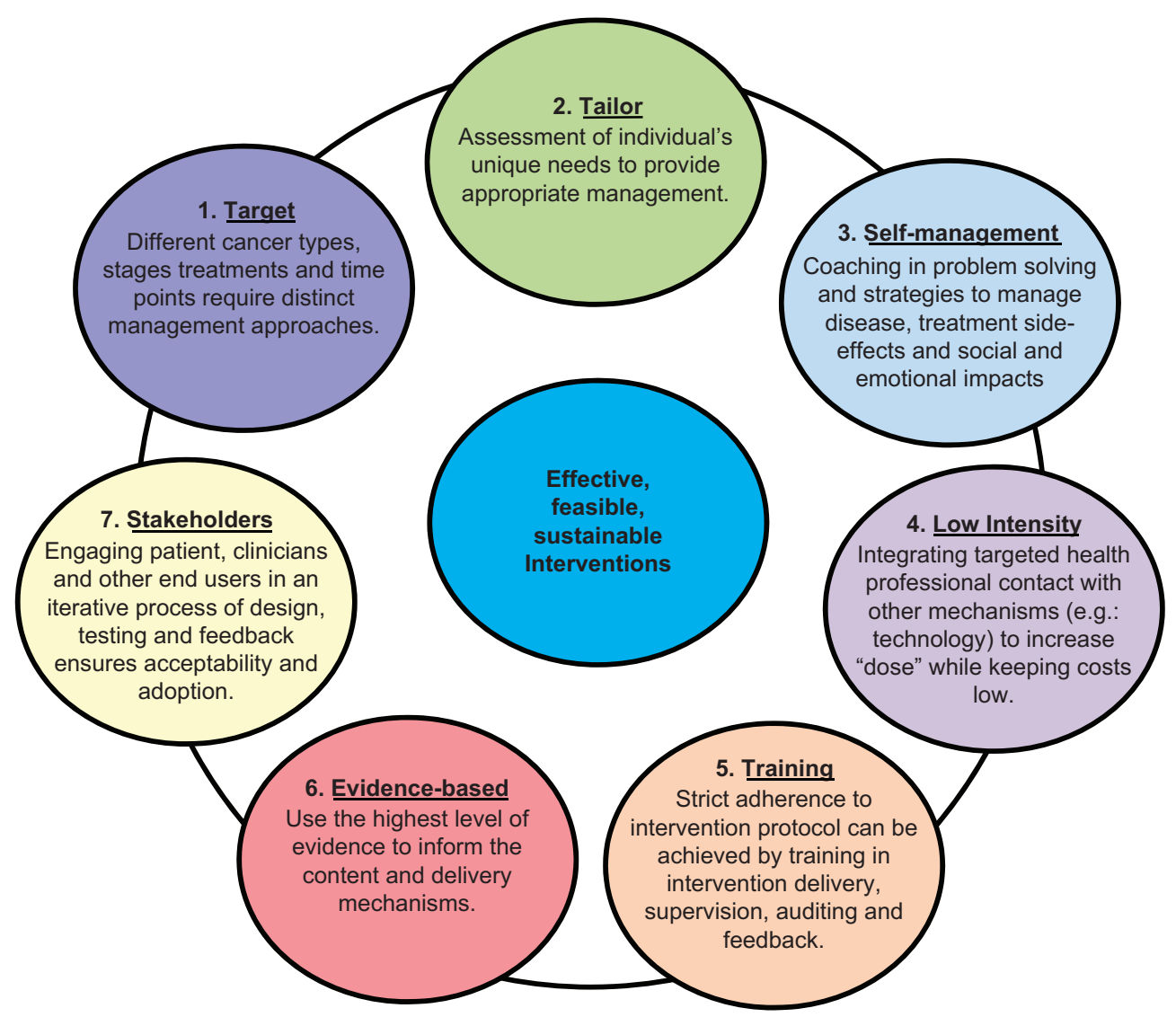

Figure 1. A framework for the development and delivery of psycho-educational and supportive care interventions.

will influence needs and direct the target of the intervention. The cultural context of the patient, carer and the community in which they live is also a central consideration and this includes the community groups with whom they self-identify. For example, cancer treatments often have a negative impact on sexuality but the nature of that impact and the effective interventions to address the impact will depend upon the type, stage, treatment and point in illness trajectory. Men with prostate cancer who receive active treatments may experience erectile dysfunction (ED) (amongst other side effects) that will require medical management (i.e. penile rehabilitation) potentially paired with sex therapy or couple counselling $[28,29]$. Problematically, many men are reluctant to seek help for this problem and treatment adherence is often poor $[29,30]$. As well, the physical effects of ED for men also have a psycho-social dimension, especially with regards to masculinity [31], and this will be a crucial consideration for intervention.

In contrast, women who have completed pelvic radiotherapy for gynaecological cancer, are at risk of vaginal-specific side effects including stenosis, agglutination, reduced genital sensations, vaginal dryness, dyspareunia, and post-coital bleeding [32]. Use of dilators, moisturisers, pelvic floor exercises and lubricants during sexual intercourse have been recommended but there is mixed evidence to demonstrate that these interventions, particularly dilator use, improve sex function or intimacy $[33,34]$. Hence, a sexuality intervention targeting this population will look very different to one designed for men with prostate cancer. With the recognition that the needs of different patient populations can vary, the first step is to understand what the distinct needs of particular patient groups are.

Tailoring to individuals' unique needs. A further consideration is tailoring the depth or intensity of intervention to need. A tiered model of care in cancer is where the depth of the intervention is tailored to each individual's level and type of need [35]. Specifically, as the level of need and complexity of the problem increases the focus of the intervention narrows and the depth of the intervention increases. Stepped care is where the intensity of the intervention is progressively stepped up until need is met, with a self-management approach potentially the first level of care $[36,37]$. For this to occur, screening for distress and need will be essential. A recent systematic review of randomised controlled trials assessing 
screening programs in cancer services indicated that systematic assessment of distress and unmet need improves doctor-patient communication and improve appropriate referral to psychosocial services [16]. Hence, systematic needs assessment has global benefits for good oncology care. Pragmatically, selfmanagement presents as a first crucial step and level of care.

Promotion of patient self-management. To shift patients from resource intensive hospital setting to the community setting, they need to be educated and coached in self-management of their disease. Interventions that provide patients with information and skills related to symptom assessment, goal setting and problem solving reduce distress, symptoms, health care use and enhance uptake of health behaviours $[25,26]$. Behavioural strategies, such as the use of question prompts and reminders, can improve communication between health professionals and patients and increase uptake of recommended behaviours [38]. Coaching in self-management and behaviour change may be accomplished using motivational interviewing which is a collaborative style of clinical interaction that elicits from the patient their own motivations to adopt a new behaviour (e.g. medication adherence) or cease an existing one (e.g. smoking) while respecting their autonomy.

Low-intensity intervention. Taking 'low intensity' approach to educating patients and their families in self-management, symptom control, behaviour change and psychological care will be more efficient than high intensity interventions and thus have greater likelihood of being broadly adopted as usual care if proved to be successful.

Low intensity approaches have been explored in the psychological domain as part of a new emerging values-based paradigm of care that has as its guiding principle improving access [39]. Taking a "low intensity" approach it would be expected that self-management for symptom management and psychosocial care would be expected to be more cost-efficient on a population level (assuming screening and referral for high need individuals) and therefore would have greater likelihood of being broadly adopted as usual care if successful. The central tenant of low intensity intervention design is that it increases access and directs the intervention "dose" where it is needed thereby using clinical services efficiently. This meets the objectives of increasing access, ensuring services are flexible and responsive to need, being patient centred, as well as cost effective.

Low intensity interventions can be achieved by the method of delivery (e.g. remote delivery via phone, internet or posted materials; group-based approaches) or applying alternative service models (e.g. use of non-specialist therapists, such as general practitioners or peers - volunteers who are cancer survivors) [40]. Increasingly these interventions rely on targeted human contact layered with technology delivered care rather than relying on either alone. Finally, intervention compatibility with existing resource infrastructure is critical so that effective interventions can be easily integrated into existing clinical services [41].

Training and adherence to protocol. Non-pharmacological, complex intervention trials require strict adherence to study protocol to ensure standard intervention delivery. The ability for clinicians to work effectively in new models of care that incorporate technology and build patients' self-management capacity relies on adequate training and ongoing supervision. A standardised manual specifying: 1) the intervention content; and 2) the training and supervision procedures are required. A random sample of intervention events should be assessed for protocol adherence to achieve quality control. These processes are also vital for dissemination into usual care and sustainability.

Evidence-based content. Interventions should be based on the best available evidence. This applies to the content of the intervention (e.g. self-management recommendations) and the mechanism of delivery (e.g. use of motivational interviewing over the phone). The evidence base must be developed if it does not exist.

Stakeholder acceptability. The intervention must also be acceptable to both patients and clinicians. Involvement of all stakeholders in the developmental phase of the intervention is critical to the uptake of the intervention. As the Medical Research Council framework [42] proposes an iterative process of designing the intervention, obtaining feedback to ensure that the intervention is relevant, acceptable and is perceived to be effective in addressing the particular needs of the target group will ensure that services resonate with the person/family's specific cancer experience and clinicians are willing to endorse the intervention.

\section{Application}

We have applied this framework to a series of randomised controlled trials, several which are now reported [40,41,43-45], with others still in progress [41,46-52]. This cycle of action and reflection has resulted in modifications to the framework and may provide insights as to why some trials may produce unexpected results. 


\section{The case of survivorship care plans: Why focus matters}

Survivorship care plans are tools aimed to ensure that cancer patient receive appropriate medical and psychosocial follow-up when treatment ends. They were a key recommendation of the Institute of Medicine report on cancer survivorship [53]. The first randomised controlled trial of the survivorship care plans was a negative trial [54]. While intervention was targeted to early-stage breast cancer, the patient population included women who were up to six years from diagnosis for whom survivorship care plans are going to be far less relevant than for those who have just completed primary treatment. Targeting of the intervention needs to incorporate point in the illness trajectory. It was also not clear if the intervention was tailored to the individual's particular circumstances or unique needs, however, there was no screening for high distress nor did the intervention incorporate self-management coaching. Patient reported outcomes are unlikely to improve unless there is a systematic assessment and addressing of needs, including enhanced self-management [55]. While it is laudable that the intervention was designed to be clinically feasible and practical, it may be that a single session was an insufficient "dose" to be effective. Other methodological issues, including floor effects, timing of primary endpoint assessment and measurement tools may also have contributed to trial results [55]. We are now testing a new intervention which incorporates survivorship care plans and is based on this framework for people completing treatment for colorectal cancer [51].

Psycho-sexual educational intervention for gynaecological cancer: Blending peer and professional help models

The following example demonstrates how the framework can be used to conceptualise and model a psycho-educational/supportive care intervention in a current ongoing randomised controlled trial where self-management is supported by both nurse led and peer support [47]. Using the proposed framework, we have developed and are testing an intervention to meet the needs of women with gynaecological cancer receiving radiotherapy by combining individually tailored nurse-led consultations with telephone-based peer support.

Radiotherapy is a common treatment for gynaecological cancer and has many distressing psychological and physical side effects. The aim of the study is to test the effectiveness of a nurse-led psychosocial intervention with peer support to reduce psychological distress, psychosocial needs, psychosexual difficulties and symptom distress, and to improve quality of life and preparation for treatment of women receiving radiotherapy with curative intent for gynaecological cancer using a randomised controlled trial.

The intervention incorporates nurse-led psychosocial sessions with telephone peer support. Peers selected are trained volunteers who are well and have completed radiotherapy for gynaecological cancer over two years prior. The nurse-led aspect involves: four consultations (pre-, mid-, end-of-treatment and two weeks post-treatment), four phone calls from a peer (pre-, mid-, end-of-treatment and four weeks post-treatment). The nurse's role was to orient the patient to treatment procedures and experiences, discuss potential short- and long-term side effects, to determine patient concerns, develop and coach patient in a self-management plan; assist the woman in psychosexual rehabilitation and coordinate multidisciplinary care referrals. The peer's role was to listen to the woman's story so far, review patient concerns and individualised self-care plan or strategies provided by the nurse; assess the woman's understanding of, and encourage adherence to, selfcare strategies, problem-solve barriers to self-care and/or encourage contact with the nurse or doctor for complex problems and encourage access to additional information and support if needed.

After completing baseline data, 306 consenting women with gynaecological cancer who are scheduled for radiotherapy are being randomised to either usual care or the intervention group. Assessments using reliable and valid questionnaires occur at baseline, pre-treatment, end of treatment, six and 12 months post-treatment.

\section{Discussion}

Over the past two decades there has been substantial progress in the development of evidence for psychosocial intervention in cancer, as demonstrated by numerous systematic reviews and meta-analyses, clinical practice guidelines, and standards for care $[41,52]$. Problematically, many trials have produced mixed results, effect sizes tend to vary, and translation of evidence-based psychosocial care into action has been less than optimal.

The evidence underpinning effective psychosocial care for men with prostate cancer is good example. Chambers and colleagues recently conducted a systematic review assessing the evidence for psychosocial interventions for men with prostate cancer [56]. There has been considerable activity in this arena with 21 trials meeting the inclusion criteria. Interventions identified as potentially effective in improving quality of life and psychological adjustment in men included group cognitive-behavioural 
and psycho-educational intervention. However, only tentative conclusions could be drawn because the evidence was mixed and overall the quality of the trials was assessed as low. It is difficult to promote uptake of interventions within health services in the absence of strong evidence particularly if there are additional costs associated with the intervention.

The present framework is offered as one way forward to address these issues and as a building block for future intervention research. We argue that the development of novel approaches to the delivery of supportive care interventions to the community to improve outcomes is a largely neglected area of major opportunity. Two key innovations here are firstly to place accessibility clearly at the centre of the intervention model; and to use the low intensity paradigm to frame this. Second, there is a need to broaden our contextualisation of care, situate the patient and carer in their community, and orient our approach accordingly. This will require movement beyond discipline and sector boundaries. The goal is clear: to address the major health problem that is the negative sequelae that accompany and follow cancer diagnosis and treatment; and the impact these sequelae have on not only the patient but also their family and carers and the community more broadly.

\section{Acknowledgements}

We would like to thank Laureate Professor Rob Sanson-Fisher for helpful comments on an early version of this framework and our collaborators on intervention studies which have assisted in shaping these concepts, in particular, Professor Sanchia Aranda and Associate Professor Michael Jefford. Our thanks also to Dr Lisa Guccione who helped finalise the paper. The authors report no conflicts of interest. The authors alone are responsible for the content and writing of the paper.

Declaration of interest: The authors report no conflicts of interest. The authors alone are responsible for the content and writing of the paper.

\section{References}

[1] Cancer IAfRo. Globocan 2012: Estimated cancer incidence, mortality and prevalence worldwide in 2012. [cited 2014 December]. Available from: http://globocan.iarc.fr/Pages/ fact_sheets_cancer.aspx 2012.

[2] Sanson-Fisher R, Girgis A, Boyes A, Bonevski B, Burton L, Cook P. The unmet supportive care needs of patients with cancer. Supportive Care Review Group. Cancer 2000;88: 226-37.

[3] Erikson C, Salsberg E, Forte G, Bruinooge S, Goldstein M. Future supply and demand for oncologists: Challenges to assuring access to oncology services. J Oncol Pract 2007;3: 79-86.
[4] Mariotto AB, Yabroff KR, Shao Y, Feuer EJ, Brown ML. Projections of the cost of cancer care in the United States: 2010-2020. J Natl Cancer Inst 2011;103:117-28.

[5] Chambers SK, Hyde MK, Au AM, Ip D, Shum D, Dunn J. A systematic review of psycho-oncology research in Chinese populations: emerging trends. Eur J Cancer Care 2013;22: 824-31.

[6] Bandura A. Self-efficacy: The exercise of control. New York: Freeman; 1997.

[7] Ajzen I. From intentions to actions: A theory of planned behavior. In: Kuhl J, Beckmann J, editors. In: Action control. SSSP Springer Series in Social Psychology: Springer Berlin Heidelberg; 1985. p 11-39.

[8] Becker MH. The health belief model and personal health behavior. Health Education Monographs 1974;2.

[9] Prochaska JO, Velicer WF. The transtheoretical model of health behavior change. Am J Health Promot 1997;12: 38-48.

[10] Graves KD. Social cognitive theory and cancer patients' quality of life: A meta-analysis of psychosocial intervention components. Health Psychol 2003;22:210-9.

[11] House JS, Kahn RL, editors. Measures and concepts of social support. New York: Academic Press; 1985.

[12] Thoits PA. Stress, coping, and social support processes: Where are we? What next? J Health Soc Behav 1995;Spec No:53-79.

[13] Dunn J, Steginga S, Rosoman N, Millichap D. A review of peer support in the context of cancer. J Psychosoc Oncol 2003;21:55-67.

[14] Deci EL, Ryan RM. Intrinsic motivation and selfdetermination in human behavior. New York: Plenum; 1985.

[15] Miller WR, Rollnick S. Motivational interviewing: Preparing people for change. New York: Guilford Press; 1991.

[16] Carlson LE, Waller A, Mitchell AJ. Screening for distress and unmet needs in patients with cancer: Review and recommendations. J Clin Oncol 2012;30:1160-77.

[17] Meijer A, Roseman M, Delisle VC, Milette K, Levis B, Syamchandra A, et al. Effects of screening for psychological distress on patient outcomes in cancer: A systematic review. J Psychosom Res 2013;75:1-17.

[18] Palmer SC, van Scheppingen C, Coyne JC. Clinical trial did not demonstrate benefits of screening patients with cancer for distress. J Clin Oncol 2011;29:e277-8; author reply e9-80.

[19] Carlson LE, Groff SL, Maciejewski O, Bultz BD. Screening for distress in lung and breast cancer outpatients: A randomized controlled trial. J Clin Oncol 2010;28: 4884-91.

[20] Hollingworth W, Metcalfe C, Mancero S, Harris S, Campbell R, Biddle L, et al. Are needs assessments cost effective in reducing distress among patients with cancer? A randomized controlled trial using the Distress Thermometer and Problem List. J Clin Oncol 2013;31:3631-8.

[21] Clark K, Bardwell W, Arsenault T, DeTeresa R, Loscalzo M. Implementing touch screen technology to enhance recognition of distress. Psychooncology 2009;18:822-30.

[22] Carter G, Britton B, Clover K, Rogers K, Adams C, McElduff P. Effectiveness of QUICATOUCH: A computerised touch screen evaluation for pain and distress in ambulatory oncology patients in Newcastle, Australia. Psychooncology 2012;21:1149-57.

[23] Clover KA, Mitchell AJ, Britton B, Carter G. Why do oncology outpatients who report emotional distress decline help? Psychooncology Epub 2014 Dec 11.

[24] Bodenheimer T, Lorig K, Holman H, Grumbach K. Patient self-management of chronic disease in primary care. JAMA 2002;288:2469-75. 
[25] Holman H, Lorig K. Patient self-management: A key to effectiveness and efficiency in care of chronic disease. Public Health Reports 2004;119:239-43.

[26] Lorig KR, Holman H. Self-management education: History, definition, outcomes, and mechanisms. Ann Behav Med 2003;26:1-7.

[27] Faller H, Schuler M, Richard M, Heckl U, Weis J, Kuffner R. Effects of psycho-oncologic interventions on emotional distress and quality of life in adult patients with cancer: Systematic review and meta-analysis. J Clin Oncol 2013; 31:782-93.

[28] Jayadevappa R, Bloom BS, Fomberstein SCKM, Wein AJ, Malkowicz SB. Health related quality of life and direct medical care cost in newly diagnosed younger men with prostate cancer. J Urol 2005;174:1059-64.

[29] Schover LR, Fouladi RT, Warneke CL, Neese L, Klein EA, Zippe C, et al. Defining sexual outcomes after treatment for localized prostate carcinoma. Cancer 2002; 95:1773-85.

[30] Miller DC, Wei JT, Dunn RL, Montie JE, Pimentel H, Sandler HM, et al. Use of medications or devices for erectile dysfunction among long-term prostate cancer treatment survivors: Potential influence of sexual motivation and/or indifference. Urology 2006;68:166-71.

[31] Chambers SK, Lowe A, Hyde MK, Zajdlewicz L, Gardiner RA, Sandoe D, et al. Defining young in the context of prostate cancer. Am J Men's Health 2014:1557988314529991.

[32] Frumovitz M, Sun CC, Schover LR, Munsell MF, Jhingran A, Wharton JT, et al. Quality of life and sexual functioning in cervical cancer survivors. J Clin Oncol 2005; 23:7428-36.

[33] Denton AS. Interventions for the physical aspects of sexual dysfunction in women following pelvic radiotherapy. Cochrane Database Syst Rev 2008.

[34] Miles T, Johnson, N. Vaginal dilator therapy for women receiving pelvic radiotherapy (Review). Cochrane Database Syst Rev 2014;9:CD007291.

[35] Hutchison SD, Steginga SK, Dunn J. The tiered model of psychosocial intervention in cancer: A community based approach. Psychooncology 2006;15:541-6.

[36] Walker S, Walker J, Richardson G, Palmer S, Wu Q, Gilbody S, et al. Cost-effectiveness of combining systematic identification and treatment of co-morbid major depression for people with chronic diseases: The example of cancer. Psychol Med 2014; 44:1451-60.

[37] van Straten A, Hill J, Richards D, Cuijpers P. Stepped care treatment delivery for depression: A systematic review and meta-analysis. Psychol Med Epub Mar 26.

[38] Dimoska A, Tattersall MH, Butow PN, Shepherd H, Kinnersley P. Can a "prompt list" empower cancer patients to ask relevant questions? Cancer 2008;113:225-37.

[39] Bennett-Levy J, Richards DA, Farrand P, Christensen H, Griffiths $\mathrm{K}$, Kavanagh D, et al. Low intensity CBT interventions: A revolution in mental health care. Oxford Guide to Low Intensity CBT Interventions. Oxford: Oxford University Press; 2010. p 3-18.

[40] Chambers SK, Girgis A, Occhipinti S, Hutchison S, Turner J, McDowell $\mathrm{M}$, et al. editors. A randomized trial comparing two low-intensity psychological interventions for distressed patients with cancer and their caregivers. Oncol Nurs Forum 2014;41:E256-66.

[41] Chambers SK, Hutchison S, Clutton S, Dunn J. Intervening to improve psychological outcomes after cancer: What is known and where next? Aust Psychol 2014;49:96-103.
[42] Craig P, Dieppe P, Macintyre S, Michie S, Nazareth I, Petticrew M. Developing and evaluating complex interventions: The new Medical Research Council guidance. London: Medical Research Council; 2008.

[43] Chambers SK, Occhipinti S, Schover L, Nielsen L, Zajdlewicz L, Clutton S, et al. A randomised controlled trial of a couples-based sexuality intervention for men with localised prostate cancer and their female partners. Psychooncology Epub 2014 Dec 8.

[44] Halkett G, O'Connor M, Aranda S, Jefford M, Shaw T, York $\mathrm{D}$, et al. Pilot randomised controlled trial of a radiation therapist-led educational intervention for breast cancer patients prior to commencing radiotherapy. Support Care Cancer 2013;21:1725-33.

[45] Schofield P, Ugalde A, Gough K, Reece J, Krishnasamy M, Carey $\mathrm{M}$, et al. A tailored, supportive care intervention using systematic assessment designed for people with inoperable lung cancer: A randomised controlled trial. Psychooncology 2013;22:2445-53.

[46] Boonzaier A, Schubach K, Troup K, Pollard A, Aranda S, Schofield P. Development of a psychoeducational intervention for men with prostate cancer. J Psychosoc Oncol 2009;27:136-53.

[47] Schofield P, Juraskova I, Bergin R, Gough K, Mileshkin L, Krishnasamy $M$, et al. A nurse and peer led support program to assist women in gynaecological oncology receiving curative radiotherapy, the PeNTAGOn study (Peer and Nurse support Trial to Assist women in Gynaecological Oncology): Study protocol for a randomised controlled trial. Trials 2013;14:39.

[48] Chambers SK, Smith DP, Berry M, Lepore SJ, Foley E, Clutton $\mathrm{S}$, et al. A randomised controlled trial of a mindfulness intervention for men with advanced prostate cancer. BMC Cancer 2013;13:89.

[49] Halkett G, O'Connor M, Aranda S, Jefford M, Spry N, Shaw T, et al. Protocol for the RT Prepare Trial: A multiplebaseline study of radiation therapists delivering education and support to women with breast cancer who are referred for radiotherapy. Br Med J Open 2014;4:e006116.

[50] Emery J, Doorey J, Jefford M, King M, Pirotta M, Hayne D, et al. Protocol for the ProCare Trial: A phase II randomised controlled trial of shared care for follow-up of men with prostate cancer. Br Med J Open 2014;4:e004972.

[51] Jefford M, Aranda S, Gough K, Lotfi-Jam K, Butow P, Krishnasamy $M$, et al. Evaluating a nurse-led survivorship care package (SurvivorCare) for bowel cancer survivors: Study protocol for a randomized controlled trial. Trials 2013; 14:260.

[52] Holland J, Watson M, Dunn J. The IPOS New International Standard of Quality Cancer Care: Integrating the psychosocial domain into routine care. Psychooncology 2011;20:677-80.

[53] Hewitt M, Greenfield S, Stovall E, editors. From cancer patient to cancer survivor: Lost in transition. Washington: National Academies Press; 2005.

[54] Grunfeld E, Julian JA, Pond G, Maunsell E, Coyle D, Folkes A, et al. Evaluating survivorship care plans: Results of a randomized, clinical trial of patients with breast cancer. J Clin Oncol 2011;29:4755-62.

[55] Jefford M, Schofield P, Emery J. Improving survivorship care. J Clin Oncol 2012;30:1391-2.

[56] Chambers SK, Pinnock C, Lepore SJ, Hughes S, O'Connell DL. A systematic review of psychosocial interventions for men with prostate cancer and their partners. Patient Educ Couns 2011;85:e75-88. 